\title{
RESULTADOS DE LASIK REALIZADO CON LÁSER DE FEMTOSEGUNDO (INTRALASE) PARA CORRECCIÓN DE MIOPÍA: EFICACIA, PREDICTIBILIDAD Y SEGURIDAD
}

\section{LASIK RESULTS OF INTRALASE FEMTOSECOND LASER TO CORRECT MYOPIA: EFFICACY, SECURITY AND PREDICTABILITY}

\author{
SÁNCHEZ-PINA JM ${ }^{1}$, ARRANZ-MÁRQUEZ E², GIL CIGANDA N ${ }^{1}$, ROMÁN GUINDO JM ${ }^{3}$, \\ TEUS MA ${ }^{4}$
}

\section{RESUMEN}

Objetivo: Evaluar los resultados refractivos del LASIK utilizando láser de femtosegundo (Intralase) para tallar el colgajo corneal, en la corrección de miopía.

Métodos: Se trata de un estudio prospectivo, observador enmascarado. Los colgajos fueron tallados con láser de femtosegundo (IntraLase). Todos los procedimientos láser fueron realizados por el mismo cirujano utilizando el láser Technolas 217 (Bausch \& Lomb). Se evaluó la agudeza visual sin corrección (AVsc) al primer día, semana, primer y tercer mes después de la cirugía, así como la agudeza visual mejor corregida (AVCC) a los tres meses de la cirugía.

Resultados: Evaluamos los resultados refractivos de LASIK en 485 ojos miopes. El defecto refractivo previo era de $-3,9 \mathrm{D}$ (DE 2,0) esfera y de $-0,9$ (DE 0,9) cilindro con una AVCC inicial de 1,1 (DE 0,1 ). Los resultados de la AVsc fueron de 0,94 (DE $0,1)$ al primer día postoperatorio, $0,96(\mathrm{DE} 0,1)$ a la

\section{ABSTRACT}

Purpose: To evaluate LASIK results obtained with the IntraLase femtosecond laser to correct myopia. Methods: This was a prospective, single masked observer study. Flaps were created with the IntraLase femtosecond laser (FS). All laser procedures were performed by the same surgeon using the Technolas 217 excimer laser (Bausch \& Lomb). We have analysed the uncorrected visual acuity (UCVA) at 1 day, 1 week, 1 month and 3 months after surgery and best spectacle-corrected visual acuity (BSCVA) after 3 months.

Results: 485 eyes with myopia were treated and their results evaluated. The mean preoperative sphere was of -3.9 D (SD 2.0) and a mean astigmatism of $-0.9 \mathrm{D}$ (SD 0.9) with BSCVA 1.1 (SD 0.1). The UCVA results were 0.94 (SD 0.1) at the first day postoperatively visit, 0.96 (SD 0.1) at first week, 1.00 (SD 0.1) at one month and 1.00 (SD 0.2) at 3 months. The refractive error at 3 months was -0.02 $\mathrm{D}$ (SD 0.3) and -0.1 D (SD 0.3) of myopia and

\footnotetext{
Recibido: 31/5/06. Aceptado: 12/6/07.

1 Diplomado en Óptica-Optometría.

2 Doctora en Medicina.

3 Licenciado en Oftalmología. Oftalmológica Madrid.

Correspondencia:

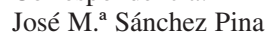

VISSUM Corporación Oftalmológica Madrid

C/. Santa Hortensia, 58

28002 Madrid

España

E-mail: jmsanchez@vissum.com
}

VISSUM Corporación Oftalmológica Madrid. Madrid. España.

${ }^{4}$ Doctor en Medicina. Profesor Titular de Oftalmología Universidad de Alcalá de Henares. Director Médico de VISSUM Corporación

Comunicación presentada parcialmente en el LXXXII Congreso de la S.E.O. (A Coruña 2006). 
semana, 1,00 (DE 0,1) al mes y $1.00(\mathrm{DE} \mathrm{0,2)} \mathrm{a} \mathrm{los}$ 3 meses. La refracción manifiesta a los 3 meses fue de -0,02 D (DE 0,3) y -0,1 D (DE 0,3) de miopía y astigmatismo respectivamente. El $96,9 \%$ de los pacientes a los 3 meses estaban dentro de un equivalente esférico de $\pm 1,00 \mathrm{D}$ y el $93,6 \%$ de los mismos se encontraban dentro de un equivalente esférico de $\pm 0,50 \mathrm{D}$.

Conclusiones: Los resultados obtenidos en nuestra experiencia parecen demostrar que el LASIK realizado utilizando láser de femtosegundo, es un método seguro, eficaz y predecible para la corrección de miopía.

Palabras clave: LASIK, Intralase, resultados, eficacia, predictibilidad y seguridad. astigmatism respectively. At 3 months $96.9 \%$ of eyes were within $\pm 1.00 \mathrm{D}$ and $93.6 \%$ of the eyes were within $+0.50 \mathrm{D}$.

Conclusions: LASIK to correct myopia is a safe, effective and predictable procedure using IntraLase FS to create the flap (Arch Soc Esp Oftalmol 2007; 82: 423-428).

Key words: LASIK, IntraLase, results, efficacy, predictability and security.

\section{INTRODUCCIÓN}

La Queratomileusis Láser In Situ (LASIK) es el procedimiento quirúrgico preferido para la corrección de error refractivo (1) gracias a su rápida rehabilitación visual y mínimo malestar postoperatorio. Esto es debido, en gran parte, a la creación de un colgajo o flap corneal que se realiza mediante microqueratomo mecánico lo que origina varias preocupaciones, como la exactitud y la reproductibilidad del grosor del mismo, además de las consiguientes complicaciones derivadas de la creación de dicho flap (2). Dicha exactitud y previsión del grosor del flap se han evaluado para la gran mayoría de los queratomos mecánicos disponibles (3-5), junto con los resultados refractivos obtenidos en LASIK utilizando dichos microqueratomos.

A pesar de los buenos resultados obtenidos, se han buscado innovaciones técnicas que igualen o superen tanto los resultados refractivos como la la creación del flap, evitando así la mayoría de sus complicaciones.

El IntraLase o láser de Femtosegundo (FS) es la primera gran alternativa al queratomo mecánico que se ha introducido comercialmente para la creación del flap en el LASIK (6).

En estudios publicados, se muestra una reducción potencial en complicaciones quirúrgicas asociadas a la creación del flap como pueden ser flaps en forma de ojales o buttonholes, flaps incompletos, y DLK, así como un alto grado de exactitud y previsibilidad de los parámetros del flap $(7,8)$.
En este estudio prospectivo, evaluamos la eficacia, predictibilidad y seguridad de la técnica LASIK utilizando el láser de Femtosegundo para la creación del flap en 485 ojos humanos con un seguimiento de 3 meses.

\section{SUJETOS, MATERIAL Y MÉTODO}

Se realiza un estudio prospectivo, observador enmascarado, sobre un total de 485 ojos sometidos a cirugía refractiva para la corrección de su defecto de graduación miópico mediante técnica LASIK utilizando el Láser de Fentosegundo (IntraLase) para la realización del flap.

Todos los pacientes fueron sometidos a una exploración oftalmológica completa que comprendía: refracción subjetiva y refracción bajo cicloplejía, topografía con el topógrafo Orbscan IIZ (Bausch\&Lomb, Rochester, NY, USA), paquimetría de contacto con el paquímetro DGH 5100, tonometría de aplanación, pupilometría medida en condiciones mesópicas con pupilómetro tipo Colvard (Oasis) y biomicroscopía.

La comprobación de la agudeza visual (AV) espontánea tras la cirugía se realizó al día siguiente, a la semana, al primer mes y al tercero, siempre por el mismo examinador, expresando los resultados en escala decimal. Para este estudio se consideraron además los resultados de la AV con corrección a los 3 meses.

La técnica quirúrgica inicial fue la siguiente: Limpieza de la piel con povidona yodada, aisla- 
miento de pestañas, colocación del blefarostato, limpieza con solución salina balanceada y marcaje con violeta de genciana.

En el IntraLase se procede posteriormente a la colocación del anillo de succión, centrándose en la córnea, hasta alcanzar una succión suficiente. Entonces la córnea es aplanada por el sistema de aplanación, eliminando todo el menisco lagrimal, realizándose el procedimiento de creación del flap. El levantamiento del flap fue retrasado durante 10 minutos, para evitar las posibles burbujas aparecidas en la interfase. Tras este tiempo se procede a la fotoablación, tras la cual el lecho estromal se irriga con solución salina balanceada y el flap es entonces recolocado de manera centrada en su lugar primitivo. Posteriormente se procede a su secado con hemostetas. Se procede igualmente al secado de la incisión con hemostetas y el procedimiento se da por concluido tras la comprobación de la correcta adhesión del flap.

Los parámetros del flap introducidos en el IntraLase para todos los pacientes fueron un espesor de flap programado a 110 micras, el ángulo de la bisagra programado a 50 grados, y la localización de todas las bisagras se realizó de modo superior. El láser usado para la fotoablación en todos los casos fue el Technolas 217C de Bausch\&Lomb, y el mismo nomograma fue adaptado para cada paciente. Todas las cirugías fueron realizadas por el mismo cirujano.

En el tratamiento postoperatorio se administró EXOCIN (ofloxacino) 1 gota 4 veces al día y MAXIDEX (dexametasona [DCI]) 1 gota 8 veces al día, durante una semana para posteriormente suspender el tratamiento.

El análisis estadístico se realizó utilizando el programa: «Statview SE+ graphics» TM (abacus concepts Inc., Cupertino, CA).

\section{RESULTADOS}

Un total de 485 ojos han sido incluidos en este estudio. La edad media ha sido de 33.4 años (DE 6,0) (rango 19-52 años). La refracción preoperatoria esférica fue de -3,9 D (DE 2,0) (rango 0 a -11D) y cilíndrica de $-0,9$ D (DE 0,9) (rango de 0 a -4,75 D). La agudeza visual mejor corregida (AVCC) media de 1,1 (DE 0,1) (rango 0,1 a 1,25) (el 94,85\% de los ojos tenían AVCC > 0,8 y el 87,62\% AVCC $\geq 1,0$ ). La zona óptica utilizada fue de $6,2 \mathrm{~mm}$ (DE 0,3 ) (rango de 5,5 a $7 \mathrm{~mm}$ ).

\section{Eficacia}

La evolución de la agudeza visual sin corrección (AVsc) después de la cirugía se muestra en la figura 1 mientras que el porcentaje de ojos que alcanzan $\mathrm{AVsc}>0,8$ y AVsc $\geq 1$ se indica en la figura 2 .

El índice de eficacia fue de 0,92.

\section{Predictibilidad}

La refracción esférica a los 3 meses postcirugía fue de -0,02 D (DE 0,3) y -0,1 D (DE 0,3) de miopía y astigmatismo respectivamente, con la que se

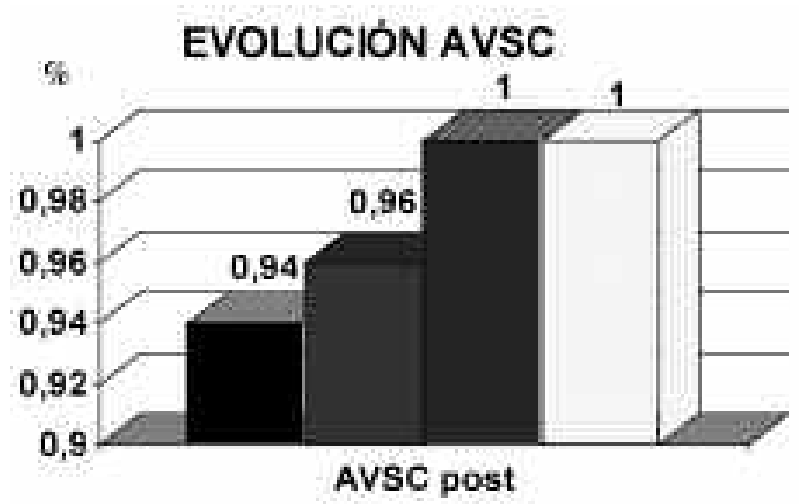

\section{1 dia 7 dias 11 mes $\square 3$ meses}

Fig. 1: Evolución de AVsc (Agudeza Visual sin corrección) tras LASIK con IntraLase al día siguiente, 7 días, 1 mes y 3 meses.

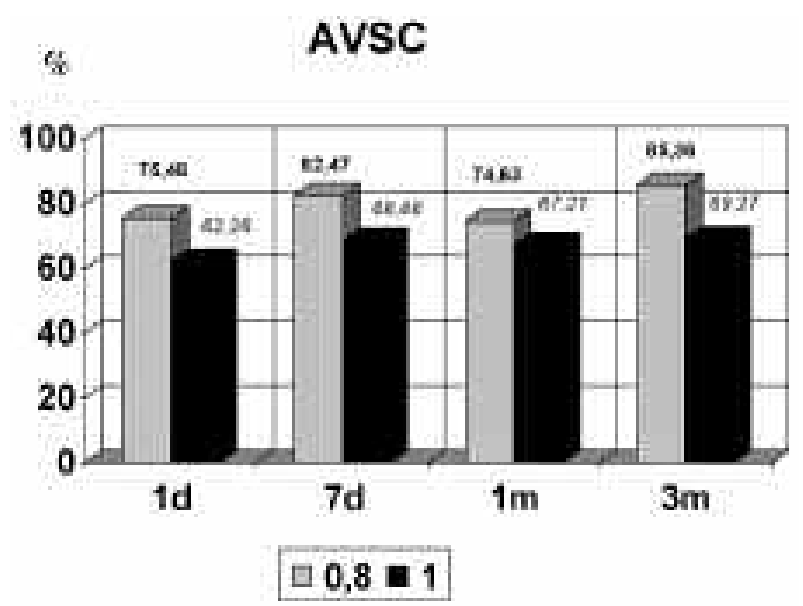

Fig. 2: Porcentaje de ojos que alcanzan AVsc > 0,8 y AVsc $\geq 1$ (agudeza visual sin corrección) tras LASIK al día siguiente, a la semana, al mes y a los tres meses. 
alcanzaba una AVCC de 1,06. Los resultados de la predictibilidad a los 3 meses vienen indicados en la figura 3.

\section{Seguridad}

El cambio de AVCC en líneas Snellen se muestra en la figura 4.

El índice de seguridad para estos pacientes fue de 0,97 .

El número de retratamientos necesarios fue de 35 ojos, lo que equivale al 7,2\% de los ojos operados.

Ninguna complicación intraoperatoria (pérdida de succión) o postoperatoria del flap (Queratitis lamelar difusa) fue observada en ningún paciente,

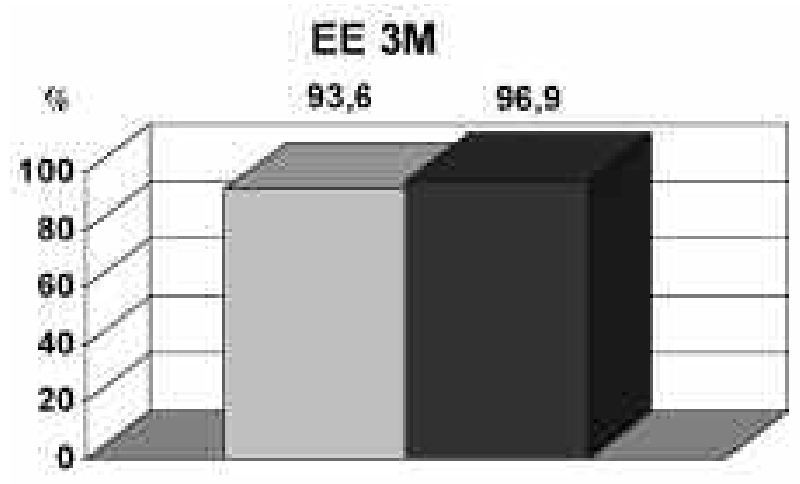

$9+1-0,50 \mathrm{EE} \mathbf{\theta}+1-1,00 \mathrm{EE}$

Fig. 3: Gráfico de barras que muestra el porcentaje de ojos dentro de un Equivalente Esférico a los 3 meses.

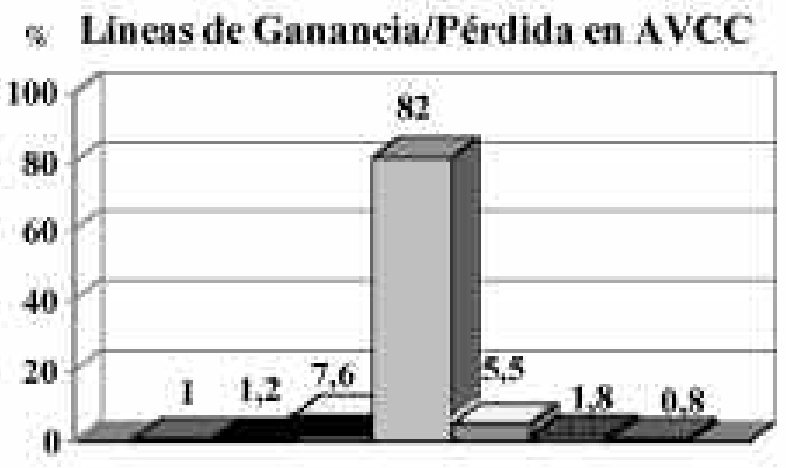

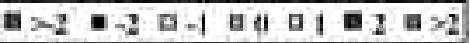

Fig. 4: Variación de líneas Snellen en AVCC (Agudeza Visual con corrección) a los tres meses. durante la creación inicial del flap o el subsiguiente levantamiento del mismo y tratamiento excímer. Un ojo presentó crecimiento epitelial $(0,2 \%)$, sin requerir nueva cirugía para su solución.

\section{DISCUSIÓN}

La reciente incorporación del láser de femtosegundo para la creación del flap en LASIK, parece ofrecer la misma seguridad y eficacia que el microqueratomo a la hora de la creación del flap, en cuanto a espesor y dimensiones $(7,8)$.

El objetivo de nuestro estudio ha sido valorar la eficacia, predictibilidad y seguridad de la cirugía LASIK utilizando esta nueva técnica de realización del flap, en 485 ojos humanos con miopía y astigmatismo miópico.

Nuestro estudio muestra que la AVsc mejora rápidamente, alcanzando valores de 1,0 al mes de la cirugía, que se mantiene a los tres meses de la misma. A los 3 meses postcirugía hemos obtenido un $85,36 \%$ de ojos con una AVsc > 0,8 y el 69,27\% de los ojos presentaron una Avsc $\geq 1$. Así encontramos que la AVsc postoperatoria era semejante a la AVCC previa a la intervención. El porcentaje de ojos que alcanzaron una $A V s c \geq 1$ en la revisión del día siguiente $(62,26 \%)$ se sitúa dentro de los porcentajes obtenidos por Kezirian (45\%) (8) y por Durrie (73\%) (9). En la revisión de la semana el 68,66\% de los LASIK de nuestro estudio alcanzaron $\mathrm{AVsc} \geq 1$ frente al $88 \%$ del trabajo de Durrie. Y esta $A V s c \geq 1$ fue alcanzada en la visita del primer mes por el $67,21 \%$ de los LASIK frente al $96 \%$ de Durrie y el $82,1 \%$ de Lim (10), mientras que en la revisión de los 3 meses nuestro dato $(69,27 \%)$ vuelve a situarse entre los obtenidos por otros estudios: $67 \%$ de Kezirian y 78,6\% de Lim. Cabe destacar que en los diferentes trabajos, el número de ojos y el equivalente esférico inicial así como el porcentaje de ojos que alcanzaban AVCC 1 previo a la cirugía eran distintos.

El porcentaje de ojos que se desviaron menos de $\pm 1,00$ D de la corrección calculada fue de $96,90 \%$ y para $\pm 0,50$ D fue del 93,60\% a los 3 meses. Kezirian et al encontraron que el $99 \%$ de los ojos se desviaban menos de $\pm 1,00 \mathrm{D}$ a los 3 meses, mientras que para $\pm 0,50 \mathrm{D}$ este dato fue del $91 \%$. Igualmente Durrie encuentra un $90 \%$ de ojos que se desviaron de $\pm 0,50 \mathrm{D}$.

En nuestra serie la refracción media postoperatoria fue de $-0,02 \mathrm{D}(\mathrm{DE} 0,3)$ de miopía y de -0,1 D 
$(0,3)$ de astigmatismo a los 3 meses, similar a la encontrada por Lim en su trabajo. El escaso valor en la refracción postoperatoria que hemos encontrado es indicativo de la estabilidad de los resultados refractivos.

La estabilidad refractiva de esta técnica ha sido confirmada por otros autores, que no encontraron pérdida importante de AVCC, significando que la técnica IntraLase tiene la misma seguridad y eficacia que la técnica con Microqueratomo. Así encontramos que en el estudio de Kezirian et al (8), a los 3 meses de la cirugía, el $2 \%$ pierde 2 filas, el $16 \% 1$ fila, mientras que el $51 \%$ mantiene la AVCC y un $33 \%$ mejoran 1 fila, datos muy parecidos a los que encuentran Nordan et al (11) con un seguimiento a 6 meses (3\% pierden 2 filas, 10\% 1 fila, el 58\% mantiene la AVCC y un $24 \%$ mejoran 1 fila). RatkayTraub (12) encuentra que a los 6 meses del LASIK, el porcentaje de ojos que perdieron 1 fila de AVCC era del 6,9\%, el 41,8\% mantenían la AVCC, mientras que ganaban 1 fila el 27,9\%, dos filas el 13,9\% y 3 o más el 9,2\%. Los datos obtenidos en nuestro estudio, reafirman estos resultados, siendo nuestro trabajo donde mayor porcentaje de ojos mantienen AVCC tras la cirugía, situándose el porcentaje de líneas perdidas en el intervalo de los trabajos citados, con la diferencia de que nuestra muestra era más grande que la de ambos estudios.

El 7,2\% de los ojos operados necesitaron un retratamiento para corregir el error refractivo residual, porcentaje similar, aunque algo inferior a los reportados por Farah et al en una revisión de la literatura $(8,2 \%$ a $17,5 \%)(13)$ y por Hersch et al, en un estudio más reciente presentan un $10,5 \%$ de incidencia de retoques (14).

La mayoría de artículos publicados sobre IntraLase comparan los resultados de LASIK obtenidos mediante láser de femtosegundo con los obtenidos utilizando microqueratomos convencionales (8-1015). Nordan et al y Ratkay-Traub presentan los resultados refractivos en LASIK realizando el tallado lamelar mediante láser de femtosegundo; de cualquier modo, todos estos trabajos presentan menos ojos estudiados que los estudiados en este artículo. Son necesarias futuras comparaciones entre los resultados obtenidos con IntraLase frente a los obtenidos con microqueratomo mecánico, con un mayor número de ojos, o similar a lo estudiado aquí.

Otros artículos sólo hablan de los parámetros del flap obtenidos con IntraLase y su comparación con los obtenidos con los actuales microqueratomos, sin reflejar los resultados refractivos obtenidos en ambos grupos (7).

A la hora de realizar el flap, la succión no se perdió en ninguno de los 485 ojos, a diferencia de otros estudios donde sí se encuentra pérdida de succión $(11,12)$. No hemos observado invasión epitelial de la interfase en el borde del flap, salvo un ojo, sin que interfiriera con la zona óptica central. Otros autores han encontrado otros tipos de complicaciones importantes con esta técnica tales como DLK (15), presencia de burbujas en cámara anterior (16), trastornos severos de sensibilidad a la luz (17)... En nuestro estudio no se presentó ninguna de estas complicaciones.

Nuestro estudio muestra que el LASIK utilizando el láser de femtoseguno o IntraLase para la realización del flap, es una técnica eficaz en la corrección de la miopía, conlleva una rápida recuperación visual, posee una gran predictibilidad y seguridad y que los resultados refractivos son estables por encima de un mes. Para confirmar que estas conclusiones favorables para el láser de femtosegundo o IntraLase se relacionan con una mayor calidad visual, más estudios y a más largo plazo son necesarios para definir el papel del IntraLase dentro de la cirugía refractiva, aunque los estudios preliminares son esperanzadores.

\section{BIBLIOGRAFÍA}

1. Sugar A, Rapuano CJ, Culbertson WW, Huang D, Varley GA, Agapitos PJ, et al. Laser in situ keratomileusis for myopia and astigmatism: safety and efficacy: a report by the American Academy of Ophthalmology. Ophthalmology 2002; 109: 175-187.

2. Stulting RD, Carr JD, Thompson KP, Waring GO 3rd, Wiley WM, Walker JG. Complications of laser in situ keratimileusis for the correction of myopia. Ophthalmology 1999; 106: 13-20.

3. Yildirim R, Aras C, Ozdamar A, Bahcecioglu H, Ozkan S. Reproducibility of corneal flap thickness in laser in situ keratomileusis using the Hansatome microkeratome. J Cataract Refract Surg 2000; 26: 1729-1732.

4. Flanagan GW, Binder PS. Precision of flap measurements for laser in situ keratomileusis in 4428 eyes. J Refract Surg 2003; 19: 113-123.

5. Pietila J, Makinen P, Suominen S, Huhtala A, Uusitalo H. Corneal flap measurements in laser in situ keratomileusis using the Moria M2 automated microkeratome. J Refract Surg 2005; 21: 377-385.

6. Sugar A. Ultrafast (femtosecond) laser refractive surgery. Curr Opin Ophthalmol 2002; 13: 246-249.

7. Binder PS. Flap dimensions created with the IntraLase FS laser. J Cataract Refract Surg 2004; 30: 26-32. 
8. Kezirian GM, Stonecipher KG. Comparison of the IntraLase femtosecond laser and mechanical keratomes for laser in situ keratomileusis. J Cataract Refract Surg 2004; 30: 804-811.

9. Durrie DS, Kezirian GM. Femtosecond laser versus mechanical keratome flaps in wavefront-guided laser in situ keratomileusis: prospective contralateral eye study. J Cataract Refract Surg 2005; 31: 120-126.

10. Lim T, Yang S, Kim M, Tchah H. Comparison of the IntraLase femtosecond laser and mechanical microkeratome for laser in situ keratomileusis. Am J Ophthalmol 2006; 141: 833-839.

11. Nordan LT, Slade SG, Baker RN, Suarez C, Juhasz T, Kurtz. R. Femtosecond laser flap creation for laser in situ keratomileusis: six-month follow-up of initial U.S. clinical series. J Refract Surg 2003; 19: 8-14.

12. Ratkay-Traub I, Ferincz, IE, Juhasz, T, Kurtz, RM, Krueger $R R$. First clinical results with the femtosecond neodyniumglass laser in refractive surgery. J Refract Surg 2003; 19: 94-103.
13. Farah SG, Azar DT, Gurdal C, Wong J. Laser in situ keratomileusis: literature review of a developing technique. J Cataract Refract Surg 1998; 24: 989-1006.

14. Hersh PS, Fry KL, Bishop DS. Incidence and associations of retreatment after LASIK. Ophthalmology 2003; 110: 748-754.

15. Tran DB, Sarayba MA, Bor Z, Garufis C, Duh YJ, Soltes $C R$, et al. Randomized prospective clinical study comparing induced aberrations with IntraLase and Hansatome flap creation in fellow eyes: potential impact on wavefront-guided laser in situ keratomileusis. J Cataract Refract Surg 2005; 31: 97-105.

16. Lifshitz T, Levy J, Klemperer I, Levinger S. Anterior chamber gas bubbles after corneal flap creation with a femtosecond laser. J Cataract Refract Surg 2005; 31: $2227-$ 2229 .

17. Stonecipher KG, Dishler JG, Ignacio TS, Binder PS. Transient light sensitivity after femtosecond laser flap creation: clinical findings and management. J Cataract Refract Surg 2006; 32: 91-94. 